\title{
Choice of summary statistics: relative and absolute measures
}

An editorial error was introduced during the preparation of this Letter for publication, which we wish to correct (BMJ 2013;346:f1092, doi:10.1136/bmj.f1092). The first sentence of the last paragraph should have read: "The findings in this review do not imply that our methods should be applied to all reviews."
Cite this as: BMJ 2013;346:f1484

๑ BMJ Publishing Group Ltd 2013 